\title{
ALTERAÇÕES NO HEMOGRAMA DE CADELAS COM NEOPLASIA MAMÁRIA
}

\author{
Antonio Henrique Cereda da Silva ${ }^{1}$, Daniella Matos da Silva ${ }^{2}$, CRistina Rauen Ribas ${ }^{3}$, \\ Rosangela LoCATElli DitTrich ${ }^{4}$, PETERSON TRICHES DORNBUSCH ${ }^{4}$, SIMONE DOMIT GuÉRIOS ${ }^{4}$ \\ ${ }^{1}$ Pós-Graduando da Universidade Federal do Paraná, Curitiba, PR, Brasil \\ ${ }^{2}$ Médica Veterinária Residente da Universidade Federal do Paraná, Curitiba, PR, Brasil - \\ dani_msufpr@yahoo.com.br \\ ${ }^{3}$ Professora Mestre da Universidade Federal do Paraná, Curitiba, PR, Brasil \\ ${ }^{4}$ Professores Doutores da Universidade Federal do Paraná, Curitiba, PR, Brasil
}

As alterações no hemograma estão direta ou indiretamente relacionadas com câncer e são pouco descritas em cadelas com neoplasia mamária. O objetivo deste estudo foi analisar as alterações hematológicas em 161 cadelas portadoras de neoplasia mamária e correlacionar à progressão da doença. As principais alterações hematológicas encontradas foram anemia normocítica e normocrômica e leucocitose neutrofílica, sendo ambas mais frequentes em cadelas em estádio avançado (III, IV ou V) da doença. Conclui-se que as alterações laboratoriais de anemia e leucocitose verificadas em cadelas com neoplasia mamária estão correlacionadas positivamente com o estádio avançado da doença.

PALAVRAS-CHAVE: anemia; estadiamento; leucocitose.

\section{COMPLETE BLOOD COUNT CHANGES IN BITCHES WITH MAMARY TUMOR}

\section{ABSTRACT}

Complete blood cell (CBC) changes are directly or indirectly related to cancer and have not been reported in bitches with mammary tumors. The aim of this study was to evaluate $\mathrm{CBC}$ changes in 161 bitches with mammary tumors, and to correlate them with disease progression.

KEYWORDS: anemia; leukocytosis; staging

\section{INTRODUÇÃO}

As neoplasias em cães e gatos têm aumentado substancialmente nos últimos anos, assumindo grande importância clínica e epidemiológica. A maior longevidade desses animais proporcionada por fatores como melhora na nutrição, cuidados na prevenção e o avanço no tratamento de doenças justificam o aumento da
Normocytic and normochromic anemia and neutrophilic leukocytosis were observed and correlated with disease stages III, IV and V. In conclusion, anemia and leukocytosis may be correlated with advanced stages of mammary tumors in bitches.

incidência de neoplasias em animais de companhia (1). Dentre os tumores espontâneos mais comuns em cães está a neoplasia mamária (2).

O papel dos hormônios sexuais no desenvolvimento dos tumores de mama em cadelas é suportado pela alta incidência em cadelas não castradas ou quando a ovariohisterectomia $(\mathrm{OH})$ é efetuada após o $2^{\circ}$ ciclo estral $(3,4)$. Clinicamente, observam-se um ou mais tumores 
palpáveis nas mamas, geralmente sem histórico de alterações sistêmicas como apatia, inapetência, febre e êmese (5).

Alterações hematológicas como anemia e trombocitopenia são relatadas em pacientes com câncer e, embora essas alterações sejam descritas na literatura veterinária, a incidência, prevalência e seu significado clínico nos animais com neoplasia são pobremente relatados (6). Anemia e coagulopatias são causas de morbidade e mortalidade em seres humanos com câncer, com impacto negativo sobre a progressão da doença e a terapia antineoplásica. Dentre os seres humanos portadores de neoplasias em grau avançado, 50 a $70 \%$ apresentam anemia (7). Quanto ao leucograma, a leucocitose é alteração importante em pacientes com neoplasia e ocorre devido à complexa interação entre as células neoplásicas com o sistema imunológico e com a inflamação peritumoral. A leucocitose neutrofílica está fortemente associada ao pior prognóstico e mortalidade em seres humanos com neoplasia, sobretudo naqueles em tratamento quimioterápico (8). No entanto, a literatura referente às alterações hematológicas em cães com neoplasia mamária é escassa e não há estudos que relacionem essas alterações com a apresentação clínica e avanço da doença.

O objetivo deste trabalho foi verificar a presença de alterações no hemograma de cadelas com neoplasia mamária, correlacionando-as com o estadiamento da doença.

\section{MATERIAL E MÉTODOS}

Foram incluídas no estudo 161 cadelas, de diferentes raças e idades, atendidas no Hospital Veterinário da Universidade Federal do Paraná (HV-UFPR) ou na Unidade Hospitalar para Animais de Companhia da Pontifícia Universidade Católica do Paraná (UHAC-PUCPR), entre os anos de 2009 e 2012, por apresentarem neoplasia mamária. Foram excluídas do estudo cadelas que apresentaram doença concomitante à neoplasia, pacientes submetidas a tratamento medicamentoso em prazo inferior a 30 dias ou animais vacinados em prazo inferior a quatro meses da data da consulta.

Durante a primeira consulta clínica das pacientes, foi colhido sangue para realização de hemograma por meio da venopunção da jugular externa. As amostras de sangue foram acondicionadas em tubos com anticoagulante ácido etilenodiaminotetracético (EDTA) e as análises hematológicas foram realizadas no Laboratório de Patologia Clínica Veterinária da Universidade Federal do Paraná. As contagens de eritrócitos, leucócitos e a determinação da concentração de hemoglobina foram realizadas no equipamento Mindray ${ }^{\circledR}$ modelo BC 2800 VET. O volume globular (hematócrito) foi realizado pela técnica do microhematócrito. $\mathrm{O}$ volume globular médio (VGM) e a concentração de hemoglobina média (CHGM) foram obtidos pelas fórmulas de Wintrobe (9). Nas extensões sanguíneas coradas com panótico rápido foram realizadas a contagem diferencial de leucócitos, as avaliações morfológicas dos eritrócitos e leucócitos e a estimativa do número de policromatófilos (policromasia).

Os valores utilizados como referência para caracterizar a anemia foram os descritos por Feldman et al. (10). O grau de anemia foi classificado pelo valor do hematócrito $(\mathrm{Ht})$, em que percentuais entre 30 e $37 \%$ indicaram anemia leve, entre 20 e $29 \%$ anemia moderada, entre 13 e $19 \%$ anemia severa e abaixo de $13 \%$ anemia muito severa. A presença de policromatófilos (policromasia) nas lâminas de esfregaço sanguíneo foi utilizada para classificar a anemia como regenerativa ou não regenerativa, considerando-se anemia não regenerativa quando $o$ grau de policromasia foi 0 (nenhum), ou raro +1 (11). Os índices hematimétricos de VGM e CHGM foram utilizados para classificar a anemia em normocítica e normocrômica. Para pesquisa de metástase e avaliação de doenças concomitantes à neoplasia mamária, as pacientes foram submetidas à radiografia torácica, em três exposições, radiografia de membros em casos de edema ou alterações oesteoarticulares e ultrassonografia abdominal exploratória. Em todas as pacientes o tecido mamário e os linfonodos regionais foram removidos cirurgicamente e submetidos à avaliação histopatológica segundo os critérios da Organização Mundial da Saúde (OMS) (12). Na presença de nódulos múltiplos foi considerado o de maior malignidade. $O$ estadiamento das pacientes seguiu a classificação TNM (tumor, linfonodo e metástase), proposto pela OMS (tabela $1)$.

As alterações hematológicas foram correlacionadas com o estadiamento da neoplasia através da aplicação do Teste Exato de Fisher, com significância de $\mathrm{P}<0,05$. 
Tabela 1 - Classificação do estadiamento clínico das neoplasias mamárias das cadelas estudadas

\begin{tabular}{lcc}
\hline Estadiamento & & Apresentação Tumoral \\
\hline I & $\mathrm{T}_{1}, \mathrm{~N}_{0}, \mathrm{M}_{0}$ & $\mathrm{~T}_{1}:$ tumor $<3 \mathrm{~cm} \varnothing$ \\
II & $\mathrm{T}_{2}, \mathrm{~N}_{0}, \mathrm{M}_{0}$ & $\mathrm{~T}_{2}:$ tumor entre $3 \mathrm{e} 5 \mathrm{~cm} \varnothing$ \\
III & $\mathrm{T}_{3}, \mathrm{~N}_{0}, \mathrm{M}_{0}$ & $\mathrm{~T}_{3}$ : tumor $>5 \mathrm{~cm} \varnothing$ \\
IV & Qualquer T, $\mathrm{N}_{1}, \mathrm{M}_{0}$ & $\mathrm{~N}_{1}$ : Linfonodo regional com envolvimento neoplásico \\
V & Qualquer T, Qualquer $\mathrm{N}_{1} \mathrm{M}_{1}$ & $\mathrm{M}_{1}$ : presença de metástases à distância \\
\hline T= tumor/N= linfonodo regional $/ \mathrm{M}=$ metástase &
\end{tabular}

\section{RESULTADOS}

A idade média das cadelas incluídas no estudo foi de 10,1 anos (5 a 17). Os animais de pequeno porte (até $10 \mathrm{~kg}$ ) representaram $41 \%$ das pacientes $(66 / 161)$, sendo a raça poodle a mais prevalente $(35 / 66)$. Os de médio porte $(10$ a $25 \mathrm{~kg})$ representaram $26 \%(42 / 161)$ e os de grande porte (mais de $25 \mathrm{~kg}), 33 \%(53 / 161)$. Dentre as pacientes do estudo, 90,1\% (145/161) eram sexualmente intactas e $9,9 \%(16 / 161)$ foram submetidas a castração tardia (após o segundo estro).

As neoplasias mamárias malignas foram mais prevalentes que as benignas, correspondendo a $87 \%(140 / 161)$ dos casos. A Tabela 2 apresenta a classificação histopatológica das neoplasias mamárias encontradas, com os respectivos números de animais acometidos.

Tabela 2 - Classificação histológica de cadelas portadoras de neoplasias mamárias de acordo com o grau de malignidade correspondente e respectivo número de animais acometidos estudados

\begin{tabular}{lc}
\hline Classificação Histopatológica & Número de casos \\
\hline Tumores Malignos & 140 \\
Adenocarcinoma Papilífero Cístico & 33 \\
Adenocarcinoma Papilífero & 22 \\
Adenocarcinoma Complexo & 22 \\
Adenocarcinoma Simples & 16 \\
Adenocarcinoma Papilífero Cístico com Áreas de Tumor Misto & 16 \\
Adenocarcinoma Sólido & 10 \\
Adenocarcinoma Complexo com Áreas de Tumor misto & 9 \\
Adenocarcinoma Tubular & 6 \\
Carcinoma Anaplásico & 2 \\
Carcinoma Esquirroso & 1 \\
Carcinoma com diferenciação Escamosa & 1 \\
Tumor misto & 1 \\
Carcinossarcoma & 1 \\
Tumores Benignos & 21 \\
Tumor Misto Benigno & 10 \\
Adenoma Simples & 4 \\
Adenoma Complexo & 3 \\
Fibroadenoma & 2 \\
Adenoma Papilífero & 1 \\
Adenoma Cístico & 1 \\
\hline Total & 161 \\
\hline
\end{tabular}

No presente estudo observaram-se $47 \%$ (76/161) dos casos diagnosticados nos estadios I $(\mathrm{n}=53)$ e II $(\mathrm{n}=23)$ e $53 \%(85 / 161)$ diagnosticados em estadios mais avançados (III/51, IV/26 e V/8). Todas as pacientes com neoplasia benigna $(n=21)$ se encontraram nos estadios iniciais (I/18 e II/3), 60\%
(85/140) das pacientes com neoplasia mamária maligna foram diagnosticadas em estadios mais avançados da doença (III/51, IV/26 e V/8). O número de casos de animais com neoplasia mamária benigna e maligna de acordo com o estadiamento da doença encontra-se na Tabela 3. 
Tabela 3 - Número de animais com neoplasia mamária benigna e maligna, de acordo com o estadiamento

\begin{tabular}{lccc}
\hline Estadiamento & Tumores benignos & Tumores malignos & $\begin{array}{c}\text { Total de casos por } \\
\text { estadiamento }\end{array}$ \\
\hline I & 18 & 35 & 53 \\
II & 3 & 20 & 23 \\
III & 0 & 51 & 51 \\
IV & 0 & 26 & 26 \\
V & 0 & 8 & 8 \\
\hline Total & 21 & 140 & \\
\hline
\end{tabular}

Ao exame físico, constatou-se em 95\% $(153 / 161)$ dos casos presença de dois ou mais nódulos em mamas, sendo que os nódulos únicos foram observados em oito pacientes com estádio I ( 5 benignos e 3 malignos). Todas as pacientes do estádio $\mathrm{V}$ apresentaram metástase pulmonar.

Alterações no hemograma foram observadas em $24,2 \%$ das pacientes $(39 / 161)$, sendo 18 animais com alterações no eritrograma, 30 no leucograma e nove em ambos. A análise do eritrograma revelou 18 $(11,2 \%)$ pacientes com anemia normocítica e normocrômica, não regenerativa. A anemia foi mais prevalente e classificada como moderada (média $\mathrm{Ht}$ $=29 \%$ ) nos animais em estadios mais avançados (14/18) quando comparado aos de estadios I e II (4/18), que tiveram a anemia classificada como leve (média $\mathrm{Ht}=34 \%$ ). $\mathrm{O}$ presente estudo revelou correlação positiva entre anemia e o avanço da doença (estadio III, IV e V; Teste Exato de Fisher,
$\mathrm{P}=0,02)$, como observado na Tabela 4 .

A análise do leucograma revelou leucocitose neutrofílica em 18,6\% (30/161) das pacientes. Nos estádios I e II, oito pacientes $(n=76)$ apresentaram leucocitose e 22 estavam nos estádios mais avançados $(n=85)$. Foi observada correlação positiva entre leucocitose e a progressão da doença (Teste Exato de Fisher, $\mathrm{P}=0,01)$, Tabela 4 . A presença de neutrofilia com desvio à esquerda ocorreu em $43 \%$ dos animais com leucocitose (13/30), sendo $10 \mathrm{em}$ estádios avançados (III, IV e V) e três em estádios I e II. A neutrofilia com desvio à esquerda não revelou correlação positiva com a progressão da doença $(\mathrm{P}>0,05$, Teste Exato de Fisher). Considerando-se apenas os pacientes com leucocitose, 30\% (9/30) apresentaram monocitose, sendo dois casos dentro dos estádios I e II e sete dentro dos estádios mais avançados.

Tabela 4 - Presença de alterações hematológicas em cadelas com neoplasia mamária de acordo com os grupos estágios iniciais (I e II) e avançados (III, IV e V) da doença

\begin{tabular}{|c|c|c|c|c|}
\hline Estadiamento & Anemia & Classificação da anemia & $\begin{array}{l}\text { Leucocitose } \\
\text { neutrofílica }\end{array}$ & $\begin{array}{l}\text { Total de casos por } \\
\text { estadiamento }\end{array}$ \\
\hline I e II & 4 & Leve (média Ht = 34\%) & 8 & 76 \\
\hline III, IV e V & 14 & $\begin{array}{c}\text { Moderada (média Ht 29\%) } \\
* \mathrm{P}=0,02\end{array}$ & $\begin{array}{c}22 \\
* \mathrm{P}=0,01\end{array}$ & 85 \\
\hline Total & 18 & & 30 & 161 \\
\hline
\end{tabular}

*Teste Exato de Fisher realizado entre os grupos

\section{DISCUSSÃO}

O presente estudo demonstrou que pacientes em estádios mais avançados da neoplasia mamária apresentaram anemia e/ou inflamação leucocitária. A anemia observada neste estudo foi leve a moderada, normocítica e normocrômica tipicamente caracterizada como anemia de doença crônica e observada em seres humanos com câncer (13). Poucos animais apresentaram alterações concomitantes no eritrograma e leucograma, por isso não foi possível correlacionar anemia com a inflamação leucocitária. Estudos futuros são necessários para verificar o papel da inflamação em cães anêmicos com neoplasia mamária.

A prevalência e relevância clínica da presença de anemia em cães com neoplasia mamária é desconhecida e pobremente reportada $(6,14)$. Entretanto, em seres humanos com câncer de mama existe uma alta incidência de anemia no diagnóstico da doença e correlação com pior resposta ao tratamento, prognóstico e sobrevida $(15,16)$. Apesar 
do baixo número de pacientes com anemia $(11,2 \%)$ neste estudo, o estádio avançado da doença está correlacionado com a presença de anemia, sugerindo que a avaliação do hemograma pode ser usada como ferramenta de determinação do prognóstico da neoplasia mamária. Cães com tumores sólidos como osteossarcoma e mastocitoma apresentam baixa incidência de anemia no momento do diagnóstico $(17,18)$, corroborando a pequena porcentagem de cães anêmicos com neoplasia mamária do presente estudo.

Quase todo distúrbio crônico com um componente inflamatório iniciará o processo que causa anemia e, entre eles, a neoplasia maligna que provoca necrose e/ou inflamação ao redor ou no interior da neoplasia (9). Na oncologia veterinária, a anemia vem sendo associada à redução na qualidade de vida, que se traduz em anorexia, perda de peso e intolerância ao exercício $(14,19)$. No presente estudo, as pacientes foram conduzidas ao serviço de oncologia por apresentarem nódulos nas mamas, sem relatos pelos proprietários de distúrbios comportamentais; no entanto, não foi realizada investigação sobre qualidade de vida e mudanças de comportamento. $\mathrm{O}$ impacto da anemia na qualidade de vida de cadelas com neoplasia mamária deve ser avaliado em estudos futuros, tendo em vista a presença da anemia em pacientes com estàdio mais avançado da doença.

A leucocitose neutrofílica verificada neste trabalho provavelmente reflete a condição inflamatória decorrente da neoplasia mamária (6). A correlação positiva da leucocitose com o estádio avançado da doença não está bem descrita na literatura, mas sugere-se que a cronicidade da neoplasia acarreta resposta inflamatória sistêmica e, por consequência, pior prognóstico. A monocitose é característica de processos inflamatórios crônicos e o aumento moderado no número de monócitos, associado ou não a quadros de neutrofilia, pode ser observado em pacientes com câncer, sobretudo em neoformações com áreas de necrose (6). Neste estudo, a monocitose associada à neutrofilia foi uma alteração hematológica presente, porém sem correlação com o estadiamento da neoplasia mamária.

Neste estudo, os tumores encontrados apresentaram diagnóstico histológico maligno em proporção maior ao relatado por trabalhos recentes brasileiros $(20,21)$, embora o diagnóstico de metástase à distância tenha sido inferior aos $25 \%$ descritos na literatura atual $(4,21)$. Esses achados provavelmente se devem ao fato das neoplasias mamárias malignas em cadelas nem sempre apresentarem características clínicas de malignidade, como foi verificado no presente trabalho, no qual
$75,7 \% \quad(106 / 140)$ das pacientes com neoplasia histologicamente maligna não apresentaram invasão tumoral e metástase, característica de tumores com comportamento benigno.

A classificação TNM, proveniente da medicina para, o estadiamento de caninos com neoplasia mamária é um fator importante a ser considerado quanto ao comportamento clínico dos tumores devido à expressiva variação individual dos animais. Como exemplo, tumor mamário maligno classificado como T1 $(<3 \mathrm{~cm})$ em um cão de pequeno porte (até $10 \mathrm{~kg}$ ) possivelmente apresentará comportamento clínico diferente em um cão de grande porte (maior que $25 \mathrm{~kg}$ ), devido à menor proporção do tumor em relação à área de superfície corporal. Dessa forma, sugere-se revisão e adequação da classificação TNM para cadelas com neoplasia mamária. É importante ressaltar que, apesar de tumores mamários pequenos $(<3 \mathrm{~cm})$ geralmente serem benignos (22), exigi-se instituição imediata de tratamento cirúrgico pois em alguns casos se comportam de forma agressiva (21).

A epidemiologia e impacto clínico das anormalidades hematológicas em cadelas com neoplasia mamária devem ser relatados, a fim de se determinar a importância de intervenção terapêutica para corrigi-las e sua influência no prognóstico da doença.

\section{CONCLUSÕES}

A anemia normocítica e normocrômica, não regenerativa, e a leucocitose neutrofílica são as alterações hematológicas mais frequentes em cadelas com neoplasia mamária estão correlacionadas positivamente com o avanço da neoplasia.

\section{COMITÊ DE ÉTICA}

O presente trabalho foi aprovado pelo comitê de ética no uso de animais (CEUA) do Setor de Ciências agrárias UFPR, campus Curitiba, sob protocolo n. 058/2011.

\section{REFERÊNCIAS}

1. De Nardi AB, Rodaski S, Sousa RS, Costa TA, Macedo TR, Rodigheri SM, Rios A, Piekarz CH. Prevalência de neoplasias e modalidades de tratamento em cães atendidos no Hospital Veterinário da Universidade Federal do Paraná. Archives of Veterinary Science. 2002; 7(2):15-26.

2. Sorenmo K. Canine mammary gland tumors. Veterinary Clinics of North America: Small Animal Practice. 2003;33:573-596.

3. Lana SE, Rutteman GR, Withrow SJ. Tumors of the 
mammary gland. In: Withrow SJ, Vail DM. Small Animal Clinical Oncology. 4th ed. St Louis: Saunders Elsevier; 2007. p. 619-636. English.

4. De Nardi AB, Rodaski S, Rocha NS, Fernandes SC. Neoplasias mamárias. In: Daleck CR, De Nardi AB, Rodaski S. Oncologia em cães e gatos. São Paulo: Roca; 2009. p. 372-383. Portuguese.

5. Sorenmo KU, Rasotto R, Zappulli V, Goldschmidt MH. Development, anatomy, histology, lynfatic drainage, clinical features, and cell differentiation markers of canine mammary gland neoplasm. Veterinary Pathology. 2011; 48(1):85-97.

6. Childress MO. Hematologic Abnormalities in the Small Animal Cancer Patient. Veterinary Clinics of North America: Small Animal Practice. 2012; 42(1):123-155.

7. Dietl B, Marienhagen J, Schäfe C, Kölbl O. The Prognostic Value of Anaemia at Different Treatment Times in Patients with Locally Advanced Head and Neck Cancer Treated with Surgery and Postoperative Radiotherapy. Clinical Oncology. 2007; 19(4):228-233.

8. Connolly GC, Khorana AA, Kuderer NM, Culakova E, Francis CW, Lyman GH. Leukocytosis, thrombosis and early mortality in cancer patients initiating chemotherapy. Thrombosis Research. 2010; 126(2):113-118.

9. Stockham SL, Scott MA.Fundamentals of Veterinary Clinical Pathology. 2nd ed. Iowa: Wiley-Blackwell; 2008. 936p. English.

10. Feldman BF, Zinkl JG, Jain CN. Schalm's Veterinary Hematology. 5th ed. Philadelphia: Lippincott Williams \& Wilkins. 2000.1344p. English.

11. Hodges J, Christopher MM. Diagnostic accuracy of using erythrocyte indices and polychromasia to identify regenerative anemia in dogs. Journal of the American Veterinary Medical Association. 2011; 238(11):14521458.

12. Meuten DJ. Tumors in domestic animals. 4th ed. Iowa: Wiley-Blackwell; 2002. 788p. English.

13. Dunst J, Kuhnt T, Strauss HG, Krause U, Pelz T, Koelbl H, Haensgen G. Anemia in cervical cancers: impact on survival, patterns of relapse, and association with hypoxia and angiogenesis. International Journal of Radiation Oncology Biology Physics. 2003; 56(3):778-
787.

14. Miller AG, Morley PS, Rao S, Avery AC, Lana SE, Olver CS. Anemia is associated with decreased survival time in dogs with lymphoma. Journal of Veterinary Internal Medicine. 2009; 23:116-122.

15. Kirshner J, Hatch M, Hennessy DD, Fridman M, Tannous RE. Anemia in stage II and III breast cancer patients treated with adjuvant doxorubicin and cyclophosphamide chemotherapy. Oncologist. 2004; 9(1):25-32.

16. Leonard RC, Untch M, Von Koch F. Management of anaemia in patients with breast cancer: role of epoetin. Annals of Oncology. 2005; 16(5):817-824.

17. Takahashi T, Kadosawa T, Nagase M, Matsunaga S, Mochizuki M, Nishimura R, Sasaki N. Visceral mast cell tumors in dogs: 10 cases (1982-1997).Journal of the American Veterinary Medical Association. 2000; 216(2):222-226.

18. Endicott MM, Charney SC, Mcknight JA, Loar AS, Barger AM, Bergman PJ. Clinicopathological findings and results of bone marrow aspiration in dogs with cutaneous mast cell tumours: 157 cases (1999-2002). Veterinary Comparative Oncology. 2007; 5(1):31-37.

19. Oyama, MA, Rush JE, O'Sullivan ML, Williams RM, Rozanski EA, Petrie J, Sleeper MM, Brown DC. Perceptions and priorities of owners of dogs with heart disease regarding quality versus quantity of life for their pets. Journal of the American Veterinary Medical Association. 2008; 233(1):104-108.

20. Oliveira Filho JC, Kommers GD, Masuda EK, Marques BMFPP, Fighera RA, Irigoyen LF, Barros CSL. Estudo retrospectivo de 1.647 tumores mamários em cães. Pesquisa Veterinária Brasileira. 2010; 30(2):177-185.

21. Ribas CR, Dornbusch PT, De Faria MR, Wouk AFPF, Cirio SM. Alterações clínicas relevantes em cadelas com neoplasias mamárias estadiadas. Archives of Veterinary Science.2012; 17(1):60-68.

22. Sorenmo MU, Kristiansen VM, Cofone MA, Shofer FS, Breen AM, Langeland M, Mongil CM, Grondahl AM, Teige J, Goldschmidt MH. Canine mammary gland tumours; a histological continuum from benign to malignant; clinical and histopathological evidence. Veterinary Comparative Oncology. 2009; 7(3):162-172. 